\title{
Workshop summary and the future
}

\author{
Gianfranco De Zotti*, Carlo Burigana ${ }^{\dagger}$ and Carlo Baccigalupi* ${ }^{* *}$ \\ * Osservatorio Astronomico di Padova, Vicolo dell'Osservatorio 5, I-35122 Padova, Italy \\ $\dagger$ ITeSRE-CNR, Via P. Gobetti 101, I-40129 Bologna, Italy \\ ${ }^{*}$ SISSA, Via Beirut 4, I-34014 Trieste, Italy
}

\begin{abstract}
We present a tentative summary of the many very interesting issues that have been addresses at this workshop, focussing in particular on the perspectives for measuring the polarization power spectra of the Cosmic Microwave Background produced by scalar and tensor perturbations, in the presence of foregrounds.
\end{abstract}

\section{INTRODUCTION}

The astonishing advances in our understanding of the basic properties of the Universe and in precision determinations of its fundamental parameters, made possible by the recent accurate measurements of acoustic peaks of the Cosmic Microwave Background (CMB) anisotropy power spectrum by the TOCO [37], Boomerang [7, 8], Maxima [20], DASI [19], and CBI [39] experiments, have strongly highlighted the extraordinary wealth of cosmological information carried by the CMB. As the ongoing MAP mission and the forthcoming PLANCK satellite will provide high sensitivity, high resolution all sky CMB temperature maps the new frontier has become CMB polarization, first investigated by [45] and [25].

The information content of the CMB polarization field is in fact richer than that of the temperature field since, in addition to amplitude it also has an orientation. Recent analyzes $[46,29,56]$ have shown that, rather than with the conventional Stokes parameters Q and U (the Stokes parameter V describing circular polarization can generally be neglected because it cannot be generated through Thomson scattering), it is more convenient to work with the two rotationally invariant fields $E$ and $B$, which are linear, but non-local, combinations of $\mathrm{Q}$ and $\mathrm{U}$. This decomposition of the $2 \times 2$ symmetric trace-free tensor describing the linear polarization state is analogous to that of a vector field into a curl and a curl-free (gradient) component (e.g. [26]).

If the CMB fluctuations are Gaussian, they can be fully characterized by four power spectra, $C_{\ell}^{T T}, C_{\ell}^{E E}, C_{\ell}^{B B}, C_{\ell}^{T E}$. Under reflections (parity transformations) $E$ transforms as a scalar (like $T$ ), while $B$ transforms as a pseudo-scalar; therefore the cross-correlations $T B$ and $E B$ vanish, as discussed by Sahzin. The only fact that the temperature field yields just the first of those power spectra indicates how CMB polarimetry increases the cosmological information. But there is much more than that: scalar perturbations, held responsible for the acoustic peaks in the temperature power spectrum, do not produce, to first order, $B$-mode signal. The $B$-mode component may contain the signature of tensor perturbations (gravity waves) produced during the inflationary epoch and allow to measure the inflaton potential height ${ }^{1}$ (see, e.g. [28])! Its detection would therefore give us a glimpse of the Universe at $10^{-38}$ seconds after the initial singularity, at energy scales of $\sim 10^{16} \mathrm{GeV}$, many orders of magnitude above those accessible at accelerators, and would therefore have profound implications not only for cosmology but also for particle physics [40].

Measurements of CMB polarization are however extremely challenging because the signal is very weak (at several $\mu \mathrm{K}$ level on small angular scales and much less on large angular scales) and liable to be strongly contaminated by polarized foreground emissions. The definition of suitable strategies therefore requires a close collaboration between experiment builders, theorists, experts on the various relevant Galactic and extra-galactic foregrounds. This very timely workshop has offered a badly needed occasion for experts in the different fields to meet.

\footnotetext{
${ }^{1}$ A $B$ component can also be generated by vector perturbations that may be excited by topological-defect models but are not present in inflationary models
} 


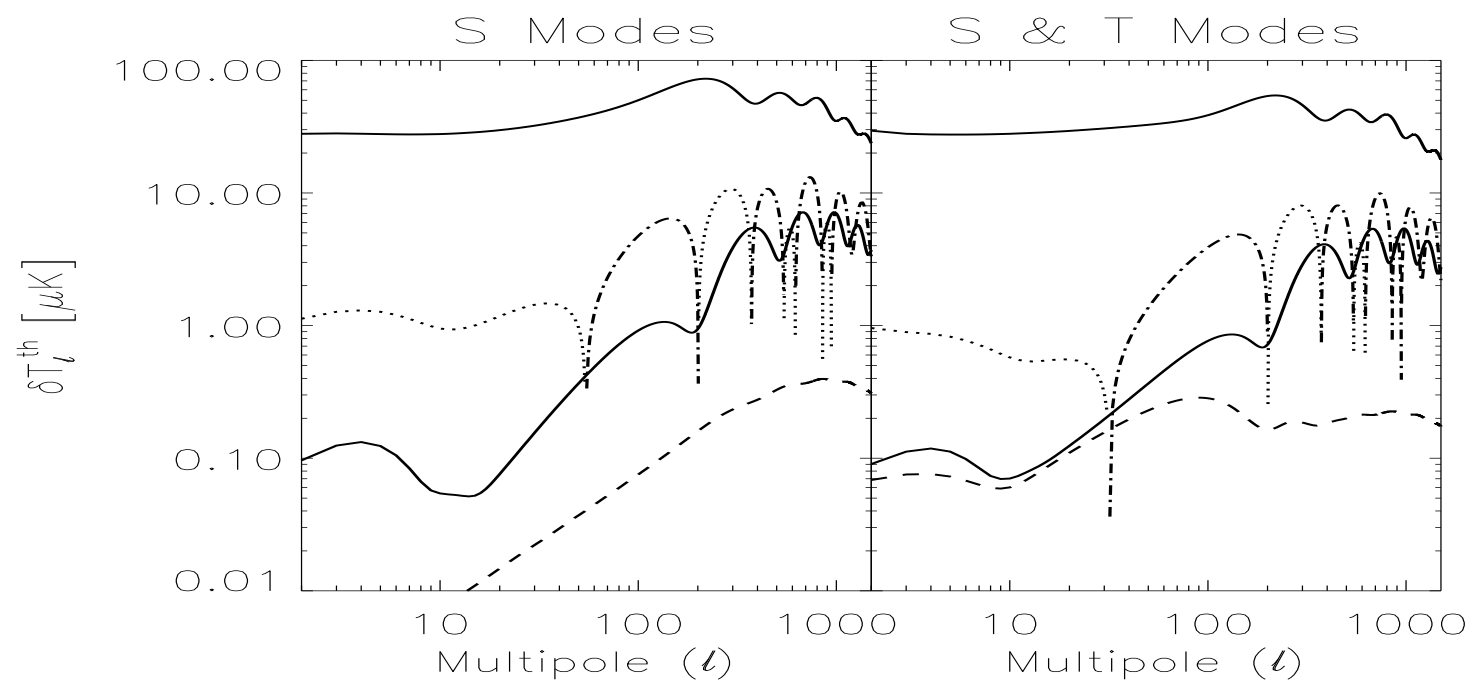

FIGURE 1. Temperature (upper solid line) and polarization power spectra for the cosmological model specified in the text. The panel on the left corresponds to pure scalar perturbations, the one on the right to scalar plus tensor perturbations yielding equal contributions to the temperature quadrupole. The partly dotted, partly dot-dashed lines represents the $T E$ cross-correlation; the dot-dashed portion shows the absolute value of the cross-correlation when it is negative. The lower solid lines and the dashed lines represent $E$ and $B$ power spectra, respectively. In the case of scalar perturbations, a $B$ contribution is originated by gravitational lensing. In the case of scalar plus tensor perturbations, gravitational lensing adds a contribution showing up at large $\ell$.

\section{CMB POLARIZATION POWER SPECTRA}

CMB polarization is induced by Thomson scattering of anisotropic radiation with a quadrupole pattern in the rest frame of the electron (see e.g. [23]). Before recombination, anisotropies were strongly damped out by the tight coupling between photons and baryons, so that the polarization that could be generated was correspondingly depressed. To generate a quadrupole, a gradient in the velocity of the photon-baryon fluid across the photon mean free path, $\lambda_{p}$, is necessary: only perturbations on scales small enough to produce anisotropies on scales $<\lambda_{p}$ can give rise to polarization. As recombination proceeds, $\lambda_{p}$ increases rapidly and polarization is produced. The polarization degree increases with decreasing angular scale (increasing $\ell$ ) reaching a maximum of $\sim 10 \%$ on the minimum scales that survived damping prior to recombination [22].

Figure 1 shows simulated temperature, polarization and $T E$ correlation power spectra (discussed by Balbi), in terms of thermodynamic temperature fluctuations per logarithmic interval in $\ell, \delta T=\left[\ell(2 \ell+1) C_{\ell} / 4 \pi\right]^{1 / 2}$, for a flat $\Lambda \mathrm{CDM}$ cosmology with $\Omega_{\text {baryon }}=0.03, \Omega_{\mathrm{CDM}}=0.3, \Omega_{\Lambda}=0.67, H_{0}=65 \mathrm{~km} \mathrm{~s}^{-1} \mathrm{Mpc}^{-1}, 3$ species of massless neutrinos, $Y_{\mathrm{He}}=0.24$ and a re-ionization optical depth $\tau=0.04$. Calculations have been made using the CMBFAST code by [47]. The panels show the cases of purely scalar, scale invariant, isentropic primordial perturbations (left), and of scalar plus tensor perturbations with a unit ratio of tensor $(T)$ to scalar $(S)$ contributions to the temperature quadrupole (right). A $T / S \simeq 1$ is probably an upper limit, once all the relevant constraints are taken into account [59].

As illustrated by Fig. 1, the ( $E$-mode) polarization fluctuations associated to acoustic peaks have relatively more power on small scales than temperature fluctuations. Also, since polarization is related to peculiar velocities on the last scattering surface, the peaks of its power spectrum are out of phase by $\pi / 2$ with those of temperature anisotropies (velocities are out of phase with density perturbations, like velocity and position of a harmonic oscillator). The $T E$ power spectrum, being the product of the two, oscillates at twice the frequency. The relative position of the temperature and polarization peaks is thus the signature of coherent perturbations (as opposed to causal mechanisms, e.g. those due to topological defects), hence of inflation, as the origin of structure. If large scale structure was produced by some causal mechanism, the first peak would have to occur at smaller angular scales in order to be within the causal horizon at last scattering [48].

A lot more can be learned from an accurate determination of the power spectrum of the $E$-mode on small angular scales, and, to some extent, to the $T-E$ correlation power spectrum $[58,31,13,14,4]$. For example, polarization measurements: identify contributions from isocurvature perturbations that can be confused with tensor perturbations or early re-ionization effects on temperature data; constrain scalar-tensor theories of gravity which may generate scalar, vector and tensor modes, leaving distinctive signatures in the CMB; are sensitive to effects of quintessence models; 
increase significantly the precision of the determination of most cosmological parameters; are directly informative on the details of the recombination and, therefore, on processes governing it [38].

As mentioned above, the amplitude of the polarization power spectrum on large angular scales originated at recombination is expected to be very small because the photon mean free path is small and multiple scattering erase polarization. On the other hand, re-ionization strongly increase the the polarization signal at low $\ell$ [55, 27] producing a characteristic bump (see Fig. 1) at $\ell \sim \sqrt{z_{\mathrm{ri}}}, z_{\mathrm{ri}}$ being the redshift of re-ionization, with amplitude roughly proportional to the optical depth for Thomson scattering. The polarization power spectrum at low $\ell$ is therefore a sensitive probe of re-ionization.

The lack of an observed Ly $\alpha$ (Gunn-Peterson) trough in the spectrum of the SDSS quasar at $z=5.8$ [17] implies, for the above choice of cosmological parameters, $\tau>0.022$. Re-ionization, however, suppresses temperature fluctuations on small scales: the lack of observed suppression of the acoustic peaks gives $\tau \lesssim 0.2$ [54], implying $z_{\mathrm{ri}} \lesssim 20$. Very recently [2] reported compelling evidence of a Gunn-Peterson trough in a $z=6.28$ quasar discovered by the Sloan survey (SDSS), suggesting that $z_{\text {ri }} \simeq 6$ (see also [16]), implying $\tau$ close to the lower limit. Keck spectroscopy by [10] have shown evidence of dark portions of the spectrum of an SDSS quasar at $z=5.73$, indicating a dramatic increase of the Ly $\alpha$ opacity at $z \gtrsim 5.2$, as expected if we are approaching (from lower redshifts) the re-ionization era of a nonuniform medium, with ionized regions intermingled with islands of neutral gas. Clearly, it may be premature to draw very definite conclusions. Only a tiny fraction $\left(\sim 2 \times 10^{-5}\right)$ of baryons need to be in the form of neutral hydrogen to account for the Gunn-Peterson optical depth implied by the data, and the re-ionization may be non-uniform, so that the line-of-sight where the effect was observed may be not representative.

On the other hand, given the rapid evolution of the optical depth that is observed, it seems unlikely that the effective re-ionization redshift is $\gg 6$. If so, the re-ionization bump shown in Fig. 1, which corresponds to $\tau=0.04$, is probably an upper limit. CMB polarization fluctuations on large angular scales are thus probably at the $0.1 \mu \mathrm{K}$ level and their detection is therefore very hard. Since the SDSS is expected to find $\sim 20$ quasars at $z \gtrsim 6$ [15], more detailed insight into the re-ionization process is expected in the next few years.

The CMB polarization power spectrum provides additional information compared to observations of the GunnPeterson trough. While the latter measures just $z_{\mathrm{ri}}$, the former also measures $\tau$, in addition to allowing an estimate of $z_{\mathrm{ri}}$ (from the position of the peak), thus providing a determination of the baryon fraction in the intergalactic medium, hence, e.g., of the galaxy formation efficiency, a piece of information very difficult to derive with other means.

The specific signature of tensor and vector perturbations is $B$-type polarization, which cannot be produced by scalar perturbations because of their symmetry properties. This opens the exciting possibility of a direct investigation of tensor components, in spite of the fact that their contribution to the CMB polarization is expected to be much smaller than that of scalar perturbations. Since the amplitude of the $B$-mode component due to gravity waves is proportional to the square of the inflationary energy scale $[56,29]$, its detection amounts to a measurement of the energy scale of inflation!

For roughly scale invariant tensor perturbations the $B$-mode power spectrum peaks at $(\ell \simeq 100$; see Fig. 1$)$. As pointed out by [57], gravitational lensing by the matter distribution, in addition to smoothing the acoustic peaks of both temperature and polarization, distorts the polarization pattern on the sky, mixing $E$ and $B$ modes. $B$-type polarization is thus generated even in the case of pure scalar perturbations. The effect peaks on small angular scales $(\ell \sim 1000)$ and does not interfere with the possibility of measuring the $B$-mode power spectrum from gravity waves.

Of course, gravity waves are not the only primary sources of $B$-mode polarization. Primordial magnetic fields (Wielebinski) would also produce tensor and vector metric perturbations, resulting in further CMB fluctuations [36, 24]. A distinctive feature of such fields is that, for a range of power spectra of perturbations produced by them, the polarization fluctuations are comparable to, or larger than, the corresponding temperature fluctuations. The amplitude of the CMB power spectra vary as the square of the energy density, i.e. as the 4th power of the magnetic field amplitude; the effects on the CMB of primordial stochastic magnetic fields with comoving amplitude much below $10^{-9}$ Gauss are essentially undetectable. Cosmological magnetic fields may also be detectable through the (frequency dependent) Faraday rotation of CMB polarization. Faraday rotation also mixes $E$ and $B$ modes. These effects are however small for realistic magnetic field strengths [33, 24].

\section{POLARIZED FOREGROUNDS}

Foregrounds may be a more serious hindrance for CMB polarization measurements than for temperature measurements because at least some of them are more polarized than the CMB. At the moment they are very poorly understood, so 


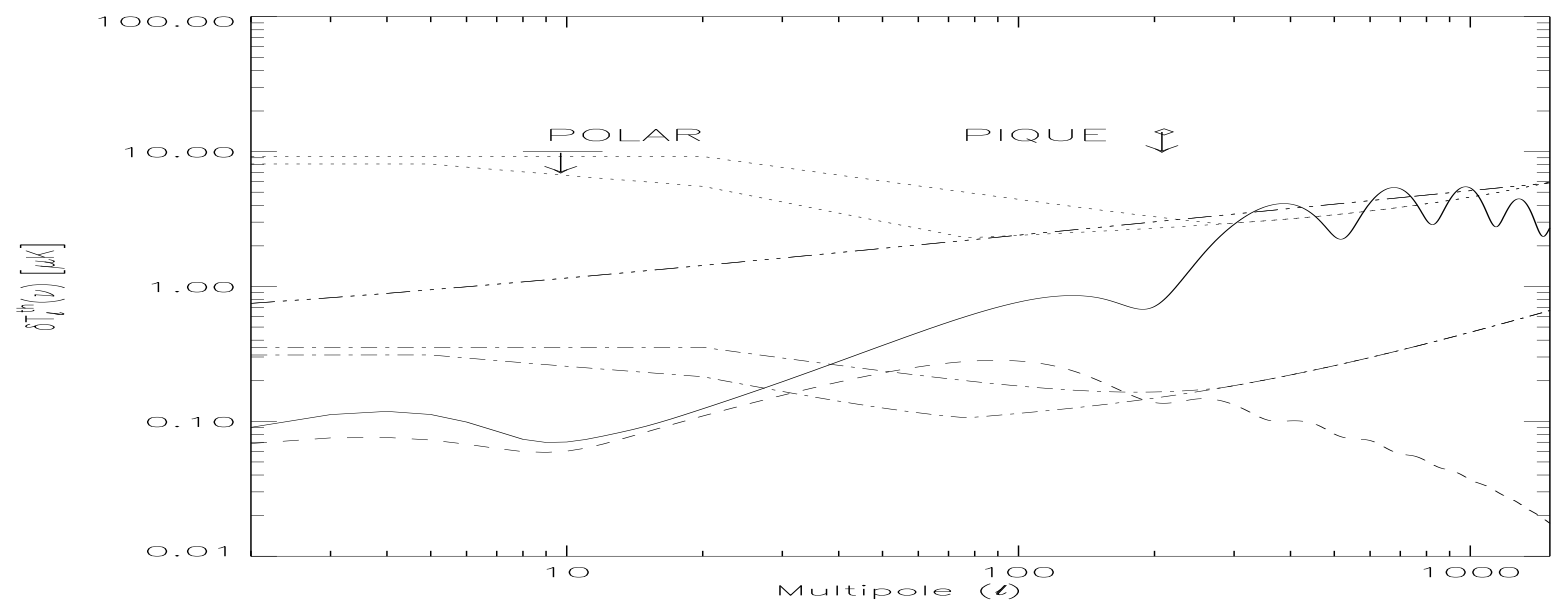

FIGURE 2. CMB versus foreground polarization power spectra. For CMB we have plotted the scalar plus tensor model of Fig. 1, but without gravitational lensing. The foreground power spectra are shown for 3 frequencies: 30 (dots), 100 (dot-dash), and 217 (three dots - dash) GHz. They comprise the contributions of Galactic synchrotron and thermal dust emission, and of extragalactic sources (radio sources and dusty galaxies); polarization fluctuations of spinning or magnetized grains are not included. At 30 and $100 \mathrm{GHz}$, the dominant polarized foregrounds are synchrotron for $\ell \lesssim 1000$ (the limit actually decreases with increasing frequency) and extragalactic radio sources at higher $\ell$. The two lines at these frequencies for $\ell \lesssim 200$ bound the range of synchrotron power spectra reported by Burigana at this meeting; the synchrotron power spectrum for higher $\ell$ is from [1]. At higher frequencies, the dominant polarized foreground is expected to be thermal dust emission, as illustrated by the line at $217 \mathrm{GHz}$. We have assumed that the global net polarization degree of dusty galaxies is $0.4 \%$, as found by Greaves (these proceedings) for M82; if so their contribution to polarization fluctuations can be significant only on very small angular scales. The $E$ and $B$ mode power spectra for these foreground components are found to be almost equal; only one of them is plotted. The recent upper limits set by the POLAR [30] and the PIQUE [21] experiments are also shown.

that it is still unclear to what extent they will limit our ability to measure CMB polarization. Particularly uncertain is the polarized emission from thermal [44], spinning and ferro- or ferri-magnetic dust grains [11].

Recent analyzes of polarization fluctuations due to the various foregrounds have been presented and discussed by several speakers (Baccigalupi, Burigana, Fosalba, Lazarian, Ponthieu, Prunet, Sazhin, Tucci). Figures 2 and 3 attempt to summarize of the main conclusions. At frequencies $\lesssim 100 \mathrm{GHz}$ foreground polarization fluctuations are likely dominated by Galactic synchrotron emission on large angular scales and up to $\ell \sim 1000$ (the limit actually decreases with increasing frequency) and by extragalactic radio sources on small scales. Note that synchrotron emission seems to have much more small-scale structure in polarization than in total intensity, at least in the relatively low-frequency maps currently available. Such structure may be due, to some extent, to differential Faraday rotation, and might therefore not be present in high frequency maps. Spinning dust grains may be important contributors particularly at $20-30 \mathrm{GHz}$, while their polarized emission is probably negligible above $40 \mathrm{GHz}$. Polarized microwave emission from magnetic grains may be important even if they do not dominate the overall emission. At higher frequencies, the main polarized foreground is expected to be, on all scales, thermal dust emission. In fact, the (still scanty) data on sub-mm polarization of dusty galaxies (Greaves, Scott) indicate that their global net polarization, is likely $<1 \%$. Polarization maps of the nearest starburst galaxy, M82 [18] show values from $0.7 \%$ to $9.1 \%$. However, when the contributions of the differently oriented magnetic vectors are summed together, the global net polarization is found to be $\simeq 0.4 \%$ (Greaves).

Figure 2 also suggests that the POLAR experiment [30], at $30 \mathrm{GHz}$, is already close to detecting synchrotron polarization fluctuations. There is apparently little hope of detecting large scale CMB polarization fluctuations at this frequency, and even at higher frequencies, a delicate subtraction of the synchrotron emission will be probably necessary. Such subtraction is further complicated by the substantial variations of the synchrotron spectral index across the sky [42].

As illustrated by Fig. 3, the foreground contamination is expected to be minimum at frequencies $\simeq 120 \mathrm{GHz}$. In the optimal frequency window, foregrounds should not seriously limit our ability to measure the $E$-mode power spectrum except for large $(\ell \lesssim 40)$ and very small $(\ell \gtrsim 2000)$ angular scales. 


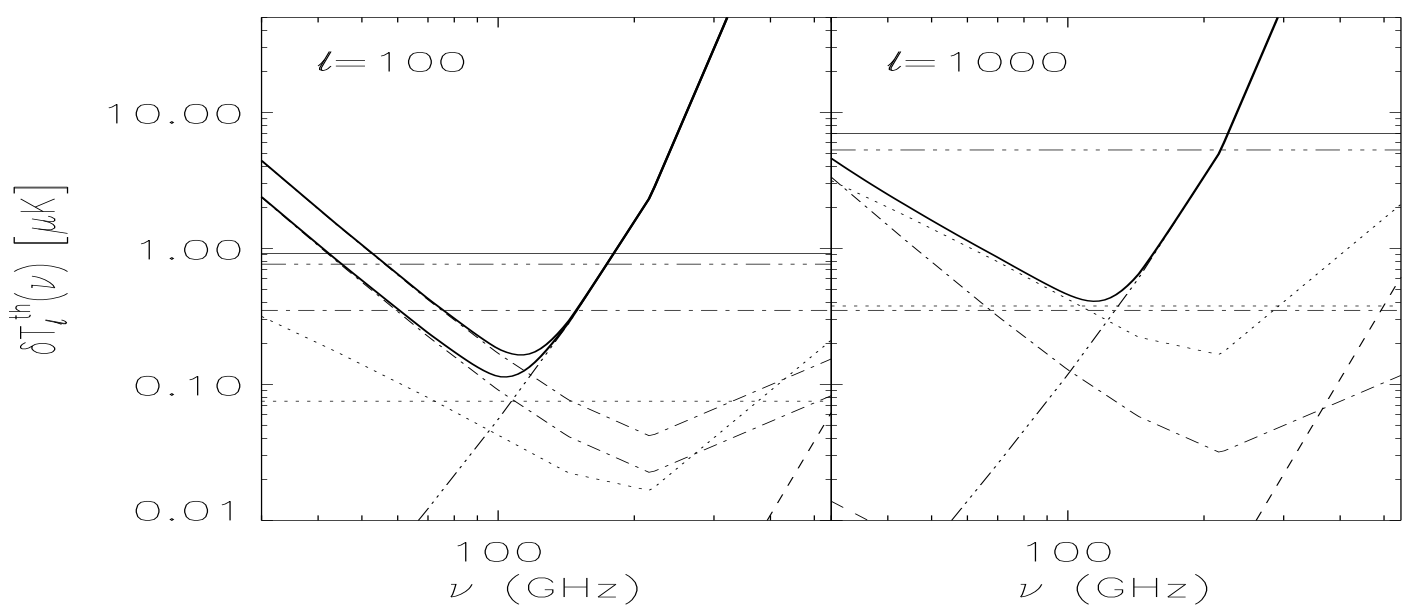

FIGURE 3. Frequency dependence of foreground polarization fluctuations for $\ell=100$ (left) and $\ell=1000$ (right). The dotdashed curves represent Galactic synchrotron. The two such curves shown in the left-hand panel bracket the estimates reported by Burigana (see caption to Fig. 2). The synchrotron curve on the right-hand panel is from [1]. The three-dots/dash curve is for thermal dust emission [44], while the dotted curve is for extragalactic radiosources [9], which, for $v \lesssim 100 \mathrm{GHz}$, dominate foreground fluctuations on small angular scales, as illustrated by the $\ell=1000$ panel. The dashed line in the right-hand corner of each panel is for dusty galaxies, assumed to have a net polarization degree of $0.4 \%$. The solid curves show the sum of the various foreground contributions. For $\ell=100$, and in general for $\ell \lesssim 200$, and $v \lesssim 100 \mathrm{GHz}$, the two solid lines reflect the range of estimates for the dominant synchrotron contributions. The horizontal lines show the CMB polarization fluctuations for the cases shown in Fig. 1, namely: $E$-mode for pure scalar fluctuations (solid), $E$-mode for pure scalar plus tensor fluctuations (three dots/dash), $B$-mode from the tensor component in the case of scalar plus tensor fluctuations (dot/dash), $B$-mode due to gravitational lensing for pure scalar fluctuations (dotted).

As pointed out by [46], in most cases foregrounds yield essentially equal contributions to the $E$ and $B$ power spectra. The analysis of low-frequency Galactic polarization surveys and of extragalactic radio sources, presented by $B a c c i g a l u p i$, indeed yields essentially equal $E$ - and $B$-mode power spectra in both cases. Since, the CMB polarization is, on small angular scales, predominantly $E$-mode, the difference between $E$ and $B$ power spectra may be a direct measure of the CMB signal.

The situation is clearly much more difficult for the $B$-mode, which, even in the optimal frequency range, appears to dominate foreground fluctuations only if the amplitude of the tensor component is close to current upper limits, and only over a rather narrow range of multipoles. Ongoing measurements and the forthcoming multifrequency maps provided by MAP and PLANCK will be essential to design future high sensitivity CMB polarization experiments.

\section{Astrophysical information from polarization surveys}

Clearly, the study of the foreground sources mentioned above is of great astrophysical interest per se, since it will provide crucial information on their physical properties. Still in the framework of CMB experiments, as noted above, some polarized astrophysical sources (Galactic synchrotron, extragalactic radio sources) may be more easily extracted from polarization than from temperature maps because their polarization degree may be higher than that of the CMB. This is particularly true for $B$-mode maps: CMB fluctuations are very small even in the "cosmological window" (50$200 \mathrm{GHz}$ ) where they dominate by far temperature maps.

High resolution, high sensitivity polarization surveys of the Galaxy are becoming available (see contributions by Reich, Gaensler, Landecker, Sault, Vinyakin), with particularly detailed imaging of the Galactic plane. These maps effectively amount to a tomography of the ISM, to quote Wielebinsky, and allow to investigate the ecosystem of the Galaxy, in Landecker's words. In fact these observations are informative on the large scale structure of the Galactic magnetic field, its structure in the solar neighborhood in relation with ISM features (Haverkorn) (cloud complexes, bubbles, loops, shells, SN remnants, etc.), its $z$-height structure and the relation between the magnetic field within the thin ISM disk and the thicker synchrotron emitting disk, the relation between the magnetic field within interstellar 


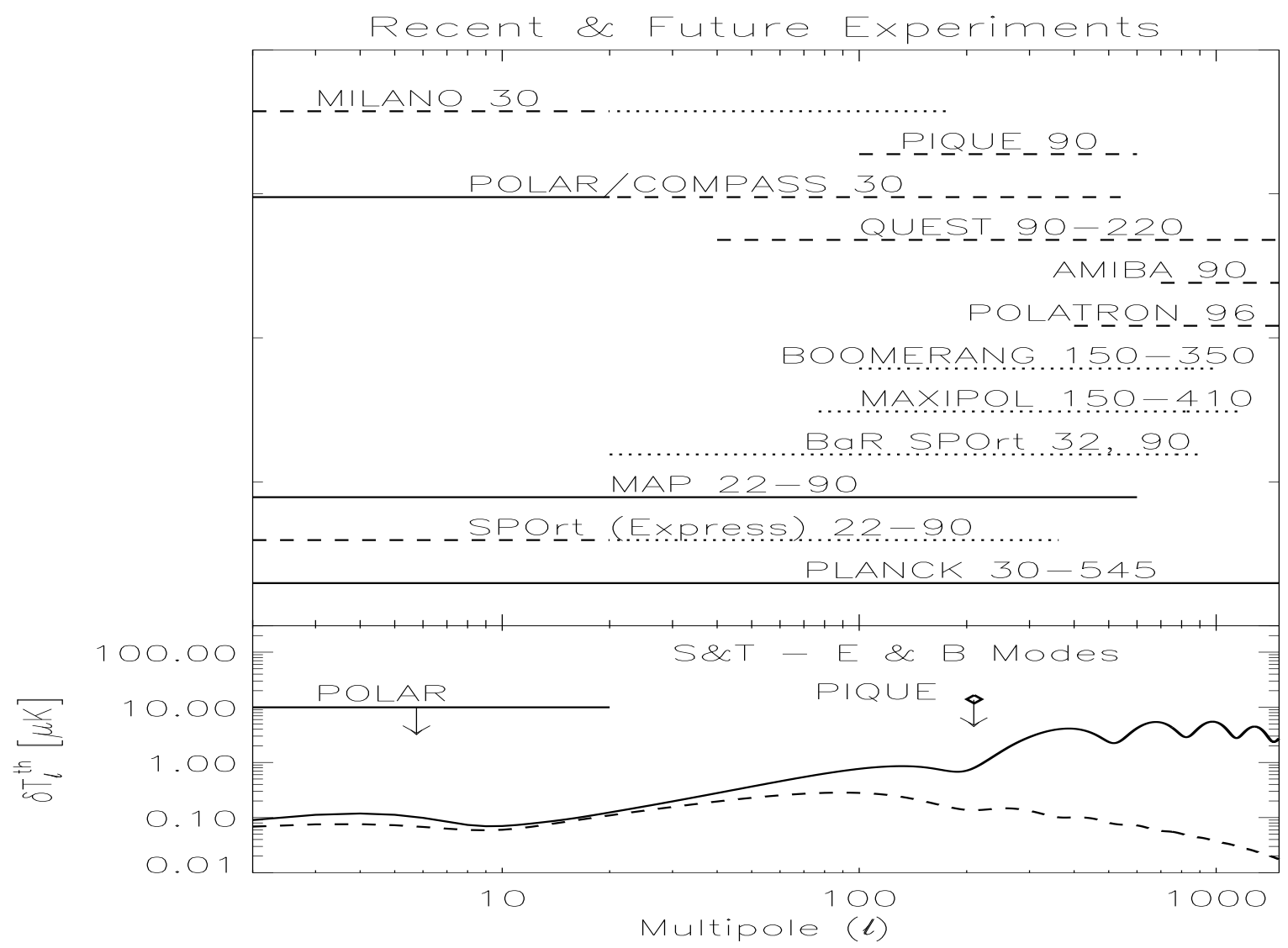

FIGURE 4. Summary of CMB polarization experiments discussed at this meeting. The straight lines show the multipole coverage. Note that the y-coordinate of such lines has nothing to do with the sensitivity of the instruments. Ground-based experiments are on top, balloon experiments in the middle and space-borne experiments on the bottom. The figures close to each experiment give the observing frequency or the frequency interval covered. The $E$ - (solid) and $B$-mode CMB power spectra (shown for reference) are for the same model as in Fig. 2.

clouds and their density and velocity structure, the role of the magnetic field in regulating the star formation efficiency, dust properties and dust alignment mechanisms (from polarization of dust emission). Our current understanding of the large scale structure of the Galactic magnetic fields has been reviewed by Han.

The available information on polarization properties of extragalactic sources is still very limited particularly at sub$\mathrm{mm}$ wavelengths (Scott, Greaves), but not much is available also for radio sources at $\gtrsim 10 \mathrm{GHz}$. There is obviously a lot of astrophysics to be learned from polarization measurements, particularly at $\mathrm{mm} / \mathrm{sub}$-mm wavelengths where the effects of internal synchrotron self-absorption and of Faraday rotation can be (with rare exceptions) ignored so that we can reliably assume that the magnetic field direction lies perpendicular to the observed polarization position angle.

Another tiny signal with a large information content is the polarized component of the Sunyaev-Zeldovich effect [50]. It will be important to assess the effect of other polarized sources either in the cluster (radio sources, radio halo $\mu$ Gauss magnetic fields and relativistic particles seem to be ubiquitous in clusters, as discussed by Wielebinski-, dust) or along the line of sight.

\section{CMB POLARIZATION EXPERIMENTS}

An excellent review of ongoing and planned CMB polarization experiments is given by [49]. Figure 4 summarizes those discussed at this meeting, indicating the multipole range they cover and the frequency bands they probe.

Although recent experiments (PIQUE [21] operating at $90 \mathrm{GHz}$, and POLAR [30] at $30 \mathrm{GHz}$ ) have considerably improved on previous results, CMB polarization is still undetected. As discussed by Timbie, the upgrade of POLAR, called COMPASS, which can reach angular scales of $20^{\prime}$, has the potential of detecting the CMB polarization if 
sensitivity per pixel will be similar to POLAR. CMB polarization measurements are expected in the next few years from several other experiments. The NASA Microwave Anisotropy Probe (MAP) is expected to obtain a highly significant detection of the CMB temperature-polarization cross-correlation, which is less affected by foregrounds than polarization maps [32]. Detection of the $E$-mode polarization is also expected from the new flights of the balloon-borne experiments, the bolometer-based, now with polarization capabilities, BOOMERANG (de Bernardis) and MAXIMA (MAXIMAPOL), and BAR-SPort (Zannoni, Macculi), using radiometers. Very promising ground based experiments include QUEST (Piccirillo) and Polatron [41], both using bolometric receivers, and the interferometer array AMiBA (Kestevens, [35]).

Measuring polarization on large angular scales (cf. the Milano experiment, presented by Gervasi, and the SPOrt project, due to fly on the International Space Station, presented by Carretti and Nicastro) appears to be much more difficult. The SPOrt-Express project, proposed for reuse of the Mars Express platform, adds to the SPOrt payload a second instrument, HARI, equipped with a 50cm antenna, giving an angular resolution $\simeq 0.5^{\circ}$ at $90 \mathrm{GHz}$.

PLANCK (see presentations by Delabrouille and Villa) should provide accurate measurements of the $E$-mode power spectrum up to $\ell \simeq 1500-2000$; its sensitivity would allow to detect $B$-mode polarization if the ratio of tensor to scalar contributions to the quadrupole anisotropy is $T / S \gtrsim 0.045$. Clearly, even if foreground contamination will not prevent us from reaching these levels, detection of $B$-mode polarization is by no means guaranteed! Much higher sensitivities are needed to explore the inflationary parameter space [26].

As discussed by Kaplan and Leahy, systematic errors may be most critical. There are a number of potential sources of systematic errors that could affect the various experiments: foreground subtraction, beam asymmetries, straylight, electronic interference, thermal variations, etc.. Experiments measuring polarization by differencing the outputs from radiometers sensitive to orthogonal polarization have common systematics for intensity and polarization measurements, although some cancellation can be expected in polarization (Leahy). The need of combining data from different detectors introduces effects of calibration, pointing, beam shape mismatches (Kaplan). Issues related to the removal of SPOrt systematics have been discussed by Amisano. Since the number of physically interesting parameters is much smaller than the number of multipoles measured in the power spectrum (typically $\sim 10$ parameters, compared with $\sim 2000$ multipoles in the case of PLANCK), very small correlated systematic errors which mimic the $\ell$-dependence of a parameter, can produce a large error in that parameter [12].

The sky coverage is also an issue. Large area maps are important to maximize the information from the power spectrum (maps of a patch of angular size $\theta$ lose the information from modes with $\ell<180^{\circ} / \theta$ ), to minimize the sample variance (which scales inversely with the sky coverage), to minimize the mixing between $B$ and $E$ caused by incomplete sky coverage (due to the non-local nature of these quantities), which may cause the $B$ component to be swamped by the, typically much larger, $E$ component [51,5]: to detect the $B$ component a survey size much larger than the coherence length of this component is required. On the other hand, given the extreme sensitivity required, very long exposures are needed to decrease the noise per pixel: with realistic integration times only relatively small patches can be covered. Optimal survey sizes may be of $\sim 20-30^{\circ}$, with $\sigma_{\text {beam }} \simeq 0.3-0.5^{\circ}[26,5,34]$.

The flood of data expected from ongoing or forthcoming CMB experiments is facing us with huge computational challenges. Balbi has described an efficient method for constructing polarization maps. Sbarra has described a new destriping technique and discussed its applications with reference to the SPOrt experiment. The application of a multifrequency Wiener filtering technique to polarized maps was investigated by [3] and [43]. The method, generalized by [52], requires the knowledge of the average frequency and angular dependence of the foreground emission. Other possibilities include pseudo- $C_{\ell}$ estimates [53] and harmonic analysis methods that bypass traditional map-based methods [6].

\section{ACKNOWLEDGMENTS}

We are grateful to the organizers of this very timely, useful and stimulating meeting. Work supported in part by ASI and MIUR.

\section{REFERENCES}

1. Baccigalupi, C., Burigana, C., Perrotta, F., et al., A\&A, 372, 8-21 (2001).

2. Becker, R.H., Fan, X., White, R.L., et al., A. J., in press, astro-ph/0108097 (2001). 
3. Bouchet, F.R., Prunet, S., Sethi, S.K., MNRAS, 302, 663-676 (1999).

4. Bucher, M., Moodley, K., Turok, N., astro-ph/ 0012141 (2000).

5. Bunn, E.F., Phys. Rev. D, submitted, astro-ph/0108209 (2001).

6. Challinor, A., et al., in preparation (2001).

7. de Bernardis, P., Ade, P., Bock, J.J., Bond, J.R., Borrill, J,, et al., Nature, 404, 955-959 (2000).

8. de Bernardis, P., Ade, P., Bock, J., Bond, J.R., Borrill, J., et al., Ap. J., in press, a st ro-ph/ 0105296 (2001).

9. De Zotti, G., Gruppioni, C., Ciliegi, P., Burigana, C., Danese, L., New Astr., 4, 481-488 (1999).

10. Djorgovski, S.G., Castro, S., Stern, D., Mahabal, A.A., Ap. J., 560, L5-L8 (2001).

11. Draine, B.T., Lazarian, A., in Microwave Foregrounds, A. de Oliveira-Costa \& M. Tegmark eds., ASP, 181, 133-149 (1999).

12. Efstathiou, G., Bond, J.R., MNRAS, 304, 75-97 (1999).

13. Eisenstein, D.J., Hu, W., Tegmark, M., Ap. J., 518, 2-23 (1999).

14. Enqvist, K., Kurki-Suonio, H., Phys. Rev. D, 61, 043002 (2000).

15. Fan, X., Narayanan, V.K., Lupton, R.H., et al., A. J., in press, astro-ph/0108063 (2001).

16. Fan, X., Narayanan, V.K., Strauss, et al., A. J., submitted, astro-ph/0111184 (2001).

17. Fan, X., White, R.L., Davis, M., et al., A. J., 120, 1167-1174 (2000).

18. Greaves, J.S., Holland, W.S., Jenness, T., Hawarden, T.G., Nature, 404, 732-733 (2000).

19. Halverson, N.W., et al.,Ap. J., in press, a stro-ph/ 0104489 (2001).

20. Hanany, S., Ade, P., Balbi, A., Bock, J., Borrill, J., et al., Ap. J., 545, L5-L9 (2000)

21. Hedman, M.M., Barkats, D., Gundersen, J.O., Staggs, S.T., Winstein, B., Ap. J., 548, L111-L114 (2001).

22. Hu, W., Dodelson, S., Ann. Rev. Astron. Ap. 2002, in press, astro-ph/ 0110414 (2001).

23. Hu, W., White, M., Ap. J., 479, 568-579 (1997).

24. Kahniashvili, T., Kosowsky, A., Mack, A., Durrer, R., in proc. Cosmology and Particle Physics, eds. J. Garcia-Pulido et al., astro-ph/0011095 (2000).

25. Kaiser, N., MNRAS, 202, 1169-1180 (1983).

26. Kamionkowski, M., Jaffe, A.H., proc. DPF2000, Columbus, in press, astro-ph/0011329 (2000).

27. Kamionkowski, M., Kosowsky, A., Phys. Rev. D, 67, 685-691 (1998).

28. Kamionkowski, M., Kosowsky, A., Ann. Rev. Nucl. Part. Sci., 49, 77-123 (1999).

29. Kamionkowski, M., Kosowsky, A., Stebbins, A., Phys. Rev. D, 55, 7368-7388 (1997).

30. Keating, B.G., O’Dell, C.W., de Oliveira-Costa, A., et al., Ap. J., 560, L1-L4 (2001).

31. Kinney, W.H., Phys. Rev. D, 58, 123506 (1998).

32. Kogut, A., and Hinshaw, G., Ap. J., 543, 530-534 (2000).

33. Kosowsky, A., Loeb, A., Ap. J., 469, 1-6 (1996).

34. Lewis, A., Challinor, A., Turok, N., Phys. Rev. D, in press, astro-ph/0106536 (2001).

35. Lo, K.Y., Chiueh, T., Liang, H., et al., in New Cosmological Data and Values of the Fundamental Parameters, IAU Symp. 201

36. Mack, A., Kahniashvili, T., Kosowsky, A., Phys. Rev. D, submitted, astro-ph/0105504 (2001).

37. Miller, A.D., Caldwell, R., Devlin, M.J., Dorwart, W.B., Herbig, T., et al., Ap. J., 524, L1-L4 (1999).

38. Naselsky, P., Schmalzing, J., Sommer-Larsen, J., Hannested, S., MNRAS, submitted, a st ro-ph/0102378 (2001).

39. Padin, S., Cartwright, J.K., Mason, B.S., Pearson, T.J., Readhead, A.C.S., et al., Ap. J., 549, L1-L5 (2001).

40. Peterson, J.B., Calstrom, J.E., Cheng, E.S., et al., a stro-ph/9907276 (1999).

41. Philhour, B.J., Keating, B.G., Ade, P.A.R., et al., a stro-ph/0106543 (2001).

42. Platania, P., Bensadoun, M., Bersanelli, M., et al., Ap. J., 505, 473-483 (1998).

43. Prunet, S., Sethi, S.K., Bouchet, F.R., MNRAS, 314, 348-353 (2000).

44. Prunet, S., Sethi, S.K., Bouchet, F.R., Miville-Deschenes, M.-A., A\&A, 339, 187-193 (1998).

45. Rees, M., Ap. J., 153, L1-L5 (2001).

46. Seljak, U., Ap. J., 482, 6-16 (1997).

47. Seljak, U., Zaldarriaga, M., Ap. J., 469, 437-444 (1996).

48. Spergel, D.N., Zaldarriaga, M., Phys. Rev. Lett., 79, 2180-2183 (1997).

49. Staggs, S.T., et al., in Microwave Foregrounds, A. de Oliveira-Costa \& M. Tegmark eds., ASP, 181, 299-309 (1999).

50. Sunyaev, R.A., Zeldovich, Ya.B., MNRAS, 190, 413-420 (1980).

51. Tegmark, M., and de Oliveira-Costa, A., Phys. Rev. D, 64, 063001-063015 (2001).

52. Tegmark, M., Eisenstein, D.J., Hu, W., de Oliveira-Costa, A., 2000, Ap. J., 530, 133-165 (2000).

53. Wandelt, B.D., Gorski, K.M., Hivon, E., in Energy Densities in the Universe, XXXV Rencontres de Moriond, astro-ph/0004178 (2000).

54. Wang, X., Tegmark, M., Zaldarriaga., M.Phys. Rev. D, in press, a stro-ph/0105091 (2001).

55. Zaldarriaga, M., Phys. Rev. D, 55, 1822-1829 (1997).

56. Zaldarriaga, M., Seljak, U., Phys. Rev. D, 55, 1830-1840 (1997).

57. Zaldarriaga, M., Seljak, U., Phys. Rev. D, 58, 023003 (1998).

58. Zaldarriaga, M., Spergel, D.N., Seljak, U., Ap. J., 488, 1-13 (1997).

59. Zibin, J.P., Scott, D., White, M., Phys. Rev. D, 60, 123513 (1999). 